\title{
Colorings and orientations of matrices and graphs
}

\author{
Uwe Schauz \\ Department of Mathematics \\ University Tübingen, Germany \\ uwe.schauz@uni-tuebingen.de
}

Submitted: Feb 9, 2005; Accepted: Jul 6, 2006; Published: Jul 28, 2006

Mathematics Subject Classifications: 05C15, 05C50, 15A15, 05C20, 05C45, 05C10

\begin{abstract}
We introduce colorings and orientations of matrices as generalizations of the graph theoretic terms. The permanent $\operatorname{per}(A[\zeta \mid \xi])$ of certain copies $A[\zeta \mid \xi]$ of a matrix $A$ can be expressed as a weighted sum over the orientations or the colorings of $A$. When applied to incidence matrices of graphs these equations include Alon and Tarsi's theorem about Eulerian orientations and the existence of list colorings. In the case of planar graphs we deduce Ellingham and Goddyn's partial solution of the list coloring conjecture and Scheim's equivalency between not vanishing permanents and the four color theorem. The general concept of matrix colorings in the background is also connected to hypergraph colorings and matrix choosability.
\end{abstract}

\section{Introduction}

The original idea behind this paper was to interpret Ryser's evaluation formula for permanents 1.2 as a statement about colorings (corollary 1.10 and the text below) and to utilize this interpretation in new proofs for Scheim's equation 2.14 and a strengthened, "quantitative" version of Alon and Tarsi's theorem 2.11. Our proofs do not use the graph polynomial, neither in combination with the combinatorial nullstellensatz as in [Al2, AlTa] nor with quantitative relations between the coefficients and the values of polynomial functions as in [Sch, Lemma 1]. The "color formula" 2.13 for $n$-regular graphs follows, unlike in [ElGo] or [Al], without use of 2-factorizations. Our methods are new and this could be of interest. However, we thought that it should be possible to use Alon and Tarsi's common and powerful methods to prove the main theorems about matrix colorings in section 1.2. This led us to the conviction that there is a stronger, "quantitative" version of the combinatorial nullstellensatz [Al2]. A paper about this stronger coefficient formula (a "combinatorial nullstellen-equation") is in preparation [Scha].

While working on this paper we realized that Alon and Tarsi's theorem $2.10 \& 2.4$ can be formulated for matrices (corollary $1.15 \& 1.6)$. Since the incidence matrix $A(\vec{G})$ 
(definition 2.1) of a directed graph $\vec{G}$ contains all information about $\vec{G}$, matrices can be seen as generalizations of directed graphs. Moreover, many graph theoretic terms (including colorings (definition 1.8) and flows) can easily be extended to matrices. Against this background it is an interesting task to formulate classical graph theoretic theorems for matrices. Our work on the Alon-Tarsi theorem is a first step in this direction. The greedy algorithm would be an other simple example. However, these investigations will have to wait for later publications.

Matrices are also connected to hypergraphs (section 3), and colorings (and nowherezero flows) of matrices are related to matrix choosability $[\mathrm{DeV}]$ and nowhere-zero points [AlTa2]. In this area the characteristic $p>0$ case is of special interest. We formulated our results for rings of characteristic 0 but that was just for simplicity; the characteristic $p>0$ case does not look much different (see also [Scha]).

This paper is structured as follows:

The general theory for matrices is developed in section 1 . We introduce the permanent and Ryser's evaluation formula and apply both, the definition and Ryser's formula, to certain copies of matrices. This leads to two types of evaluation formulas for the permanent of copies of matrices $A$. One in terms of certain orientations of $A$, the other in terms of colorings of $A$. First, in section 1.1, orientations of matrices are defined and discussed. The matrix polynomial, a generalization of the graph polynomial, is introduced here, too. Then, in section 1.2, colorings are defined and evaluation formulas are given in various degrees of generality.

In section 2 we specialize our results to the graph-theoretic situation. First, in section 2.1, orientations, Eulerian subgraphs and the graph polynomial are discussed. Then, in section 2.2, vertex colorings are introduced and a new proof of Alon and Tarsi's theorem emerges. Finally, in section 2.3, we further specialize our results to line graphs of $n$-regular and planar $n$-regular graphs. This leads us to Scheim's expression for the number of edge $n$-colorings of a $n$-regular planar graph as a permanent and to Ellingham and Goddyn's partial solution of the list coloring conjecture.

Following the referee's suggestion, we included an additional section 3 about hypergraph colorings. We are grateful for this and other helpful comments by the referee.

\section{Matrices}

Notation. In this section $V, \bar{V}, \tilde{V}$ and $E, \bar{E}$ stand for finite sets. $R$ is an integral domain of characteristic 0 (i.e. $\mathbb{Z} \subseteq R$ ). For tuples $a=\left(a_{v}\right)_{v \in V} \in R^{V}$ we write:

$V, E$

$\Pi a=\Pi\left(a_{v}\right)_{v \in V}:=\prod_{v \in V} a_{v} \quad$ and $\quad \Sigma a=\Sigma\left(a_{v}\right)_{v \in V}:=\sum_{v \in V} a_{v}$. $V \uplus \bar{V}$ denotes the disjoint union of $V$ and $\bar{V}$ (e.g. $|V \uplus V|=2|V|)$.

For $\varphi: V \longrightarrow E, \bar{\varphi}: \bar{V} \longrightarrow \bar{E}$ the map $\varphi \uplus \bar{\varphi}: V \uplus \bar{V} \longrightarrow E \cup \bar{E}$ is the union of $\varphi \subseteq V \times E$ and $\bar{\varphi} \subseteq \bar{V} \times \bar{E}$ with $V$ and $\bar{V}$ regarded as disjoint (it is again a map).

Definition 1.1 (Permanent). Let $A=\left(a_{e v}\right) \in R^{E \times V}$ be square.

The sum over all diagonal-products of $A$ is called permanent of $A$ :

$$
\operatorname{per}(A):=\sum_{\substack{\psi: E \rightarrow V \\ \text { bijective }}} \Pi\left(a_{e, \psi_{e}}\right)_{e \in E}
$$


Note that the determinant $\operatorname{det}(A)$ of the "matrix" $A$ is not defined since the determinant is not invariant under permutations of rows and columns and there are (in general) no distinguished orderings on the columns and the rows of $A$ (and also no special bijection between them). $A$ is not actually a matrix in that stronger sense.

Beside that difference in generality the permanent is a relative of the determinant and they have many properties in common. The permanent is multilinear in the columns and the rows, it is invariant by transposition of the matrix and the Laplace expansion works the same, except that you do not have to consider different signs. But there are also some main differences. The product theorem does not hold for permanents and it is not invariant under the elementary row and column operations. This deficiency makes the evaluation of the permanent difficult. A simple consequence of the principle of inclusion and exclusion and one of the best evaluation methods is the formula of Ryser [BrRy, p.200] [Mi, p.124], which we consider for more theoretical reasons:

Theorem 1.2 (Formula of Ryser). Let $A \in R^{E \times V}$ be square.

$$
\operatorname{per}(A)=\sum_{d \in\{0,1\}^{V}}(-1)^{\left|d^{-1}(0)\right|} \Pi(A d) .
$$

In what follows we investigate the permanent of certain copies $A[\zeta \mid \xi]$ of $A$ using this two formulas, where copies are defined as follows:

Definition 1.3 (Copier). Let $A=\left(a_{e v}\right) \in R^{E \times V}$ be given.

A (not necessarily surjective) map $\xi: \bar{V} \longrightarrow V, u \longmapsto \xi_{u}$ with codomain equal to the set $V$ of column indices of $A$ is a column copier to $A$, a map $\zeta: \bar{E} \longrightarrow E$ with codomain equal to the set of row indices $E$ of $A$ is a row copier to $A$.

$A[\zeta \mid \xi]:=\left(a[\zeta \mid \xi]_{e v}\right)_{e \in \bar{E}, v \in \bar{V}} \quad$ with $\quad a[\zeta \mid \xi]_{e v}:=a_{\zeta e, \xi_{v}}$ is the $(\zeta, \xi)$-copy of $A$.

We abbreviate $A[\mid \xi]:=A[\operatorname{Id} \mid \xi]$ and so on.

$\zeta$ and $\xi$ are said to be a (square) pair of copiers to $A$ if $A[\zeta \mid \xi]$ is a square matrix, i.e. if $|\bar{E}|=|\bar{V}| . \zeta$ is square if $A[\zeta \mid]$ is square, i.e. if $|\bar{E}|=|V| . \xi$ is square if $A[\mid \xi]$ is square, i.e. if $|\bar{V}|=|E|$.

\subsection{Orientations and Realizations}

In this section we evaluate $\operatorname{per}(A[\zeta \mid \xi])$ by definition 1.1 in terms of orientations.

Definition 1.4 (Orientation, Realization). Let $A=\left(a_{e v}\right) \in R^{E \times V}$ be given.

A map $\varphi: E \longrightarrow V, e \longmapsto \varphi_{e}$ with $\pi_{A}(\varphi):=\Pi\left(a_{e, \varphi_{e}}\right)_{e \in E} \neq 0$ is an orientation of A. $D(A)$ denotes the set of orientations of $A$.

We set $\left|\varphi^{-1}\right|:=\left(\left|\varphi^{-1}(v)\right|\right)_{v \in V}$ for maps into $V$. The orientation $\varphi$ of $A$ is a realization (in $A$ ) of a column copier $\xi: \bar{V} \rightarrow V$ of $A$ if $\left|\varphi^{-1}\right|=\left|\xi^{-1}\right|$. It is a realization (in $A$ ) of $\delta \in \mathbb{N}^{V}$ if $\left|\varphi^{-1}\right|=\delta . D_{\delta}(A)$ denotes the set of realizations of $\delta \in \mathbb{N}^{V}$ in $A$.

$\pi_{A}(\varphi)$

$D(A)$

$\left|\varphi^{-1}\right|$

$D_{\delta}(A)$

Theorem 1.5 ( $\pi$-formula). Let $\zeta: \bar{E} \rightarrow E$ and $\xi: \bar{V} \rightarrow V$ be a pair of copiers to $A \in R^{E \times V}$.

$$
\operatorname{per}(A[\zeta \mid \xi])=\left|\xi^{-1}\right| ! \quad \sum_{\substack{\varphi: \bar{E} \rightarrow V \\\left|\varphi^{-1}\right|=\left|\xi^{-1}\right|}} \pi_{A[\zeta \mid]}(\varphi) \text { with }\left|\xi^{-1}\right| !:=\prod_{v \in V}\left(\left|\xi^{-1}(v)\right| !\right)
$$


Proof. Let $\Psi:=\{\psi: \bar{E} \rightarrow \bar{V} \quad \mid \quad \psi$ bijective $\}$ and $\Phi:=\left\{\varphi: \bar{E} \rightarrow V \quad\left|\varphi^{-1}\right|=\left|\xi^{-1}\right|\right\}$. We compare the summands in $\operatorname{per}(A[\zeta \mid \xi])=\sum_{\psi \in \Psi} \pi_{A[\zeta \mid \xi]}(\psi)$ with those in $\sum_{\varphi \in \Phi} \pi_{A[\zeta \mid]}(\varphi)$. If $\varphi=\xi \circ \psi$ then $\pi_{A[\zeta \mid \xi]}(\psi)=\pi_{A[\zeta]]}(\varphi)$. Since the map $\psi \longmapsto \xi \circ \psi, \Psi \longrightarrow \Phi$ is surjective and each $\varphi \in \Phi$ has exactly $\left|\xi^{-1}\right|$ ! preimages $\psi \in \Psi$ (for all $v \in V$ the bijection $\psi$ has to map $\varphi^{-1}(v)$ onto $\xi^{-1}(v)$ and there are $\left|\xi^{-1}(v)\right|$ ! ways to do this) we have a $\left|\xi^{-1}\right|$ ! to 1 correspondents between the summands, and the theorem follows.

Corollary 1.6. Let $\xi: \bar{V} \rightarrow V$ be a square column copier to $A \in R^{E \times V}$.

$$
\operatorname{per}(A[\mid \xi])=\left|\xi^{-1}\right| ! \sum_{\varphi \in D_{\left|\xi^{-1}\right|}(A)} \pi_{A}(\varphi)
$$

Especially, $\operatorname{per}(A[\mid \xi])=0$ if $\xi$ does not have any realizations $\left(D_{\left|\xi^{-1}\right|}(A)=\varnothing\right)$.

The polynomial $f_{A}$ defined below is considered by many authors. In connection with the combinatorial nullstellensatz it could be used for a proof of 1.15 and 2.11 as in $[\mathrm{AlTa}]$. We want to name it matrix polynomial since it is a generalization of the graph polynomial $f_{\vec{G}}$ (see proposition 2.2). The product $A X$ in the definition of $f_{A}$ is the standard matrix-tuple product over the ring $R[X]$. We use the standard multiindex notation, $X^{\delta}:=\prod_{v \in V} X_{v}^{\delta_{v}}$ and $\delta !:=\prod_{v \in V}\left(\delta_{v} !\right)$ for $\delta \in \mathbb{N}^{V}$. The expression with the permanents has also been used in [AlTa2, Claim 1].

Definition 1.7 (Matrix polynomial). Assume $A=\left(a_{e, v}\right) \in R^{E \times V}$, let $X=\left(X_{v}\right)_{v \in V}$ be a tuple of indeterminacies. For each $\delta \in \mathbb{N}^{V}$ with $D_{\delta}(A) \neq \varnothing$ choose a $\xi^{\delta} \in D_{\delta}(A)$.

$$
f_{A}(X):=\Pi(A X) \stackrel{1.4}{=} \sum_{\varphi \in D(A)} \pi_{A}(\varphi) X^{\left|\varphi^{-1}\right|} \stackrel{1.6}{=} \sum_{\substack{\delta \in \mathbb{N} V \\ D_{\delta}(A) \neq \varnothing}} \frac{1}{\delta !} \operatorname{per}\left(A\left[\mid \xi^{\delta}\right]\right) X^{\delta}
$$

\subsection{Colorings}

Here we use the formula of Ryser 1.2 to work out $\operatorname{per}(A[\zeta \mid \xi])$ in terms of colorings.

Definition 1.8 (Coloring). Let $A \in R^{E \times V}$ be given.

A map $c: V \longrightarrow R\left(c \in R^{V}\right)$ is a coloring of $A$ if $\Pi(A c) \neq 0$.

Theorem 1.9 (Simple color formula). Let $\zeta: \bar{E} \rightarrow E$ and $\xi: \bar{V} \rightarrow V$ be a pair of copiers to $A \in R^{E \times V}$ and $Z:=\left(Z_{u}\right)_{u \in \bar{V}}, X=\left(X_{e}\right)_{e \in \bar{E}}$ tuples of indeterminacies.

Define $\bar{v}:=\xi^{-1}(v)$ and $C_{\bar{v}}:=\left\{\sum_{u \in \bar{v}} d_{u} Z_{u}: d \in\{0,1\}^{\bar{v}}\right\} \subseteq \mathbb{Z}[Z] \subseteq R[Z]$ for $v \in V$ and let $C_{\xi}(Z):=\left\{c: V \ni v \mapsto c_{v} \in C_{\bar{v}}\right\}=\prod_{v \in V} C_{\bar{v}} \subseteq R[Z]^{V}$ be the set of maps that assign an "abstract" color $c_{v}$ from the list $C_{\bar{v}}$ to each column $v \in V$.

$$
\operatorname{per}(A[\zeta \mid \xi]) \Pi Z=(-1)^{|\bar{V}|} \sum_{c \in C_{\xi}(Z)}(-1)^{\Sigma \hat{c}} \Pi(A[\zeta \mid] c-X) \quad \text { with } \hat{c}:=\left(c_{v}(1,1, \ldots, 1)\right)_{v \in V} \text {. }
$$


Proof. We multiply each column $u \in \bar{V}$ of $A[\zeta \mid \xi]$ with $Z_{u}$ and get a matrix $B=$ $\left(a[\zeta \mid \xi]_{e u} Z_{u}\right)_{e \in \bar{E}, u \in \bar{V}}$. From this we construct a matrix $C$ that is one column $u^{\prime}$ and one row $e^{\prime}$ bigger. We write "under" each column of $B$ a 0 and "behind" each row $e \in \bar{E}$ the term $-X_{e}$, on the remaining position "bottom right" we put a 1 . On the "diagonal" of this matrix there are two square blocks $B$ and the 1, "below" are only zeros, it has therefore the same permanent as $B: \operatorname{per}(C)=\operatorname{per}(B)=\operatorname{per}(A[\zeta \mid \xi]) \Pi Z$.

On the other hand the permanent of $C$ can be evaluated by the formula of Ryser 1.2 :

$$
\operatorname{per}(C) \stackrel{1.2}{=} \sum_{d \in\{0,1\}^{\bar{V} \uplus\left\{u^{\prime}\right\}}}(-1)^{\left|d^{-1}(0)\right|}\left[\prod_{e \in \bar{E}}\left(\left(\sum_{u \in \bar{V}} a[\zeta \mid \xi]_{e u} Z_{u} d_{u}\right)-X_{e} d_{u^{\prime}}\right)\right] \cdot 1 d_{u^{\prime}}
$$

since only summands to $d \in\{0,1\}^{\bar{V} \uplus\left\{u^{\prime}\right\}}$ with $d_{u^{\prime}}=1$ are $\neq 0$ this is

$$
=\sum_{d \in\{0,1\} \bar{V}}(-1)^{\left|d^{-1}(0)\right|} \prod_{e \in \bar{E}}\left(\left(\sum_{u \in \bar{V}} a[\zeta \mid \xi]_{e u} Z_{u} d_{u}\right)-X_{e}\right)
$$

now $\left|d^{-1}(0)\right|=|\bar{V}|-\left|d^{-1}(1)\right|=|\bar{V}|-\sum_{u \in \bar{V}} d_{u}$ gives

$$
\begin{aligned}
& \stackrel{1.3}{=} \sum_{d \in\{0,1\}^{\bar{V}}}(-1)^{|\bar{V}|-\sum_{u \in \bar{V}} d_{u}} \prod_{e \in \bar{E}}\left(\left(\sum_{u \in \bar{V}} a[\zeta \mid]_{e, \xi_{u}} Z_{u} d_{u}\right)-X_{e}\right) \\
& =(-1)^{|\bar{V}|} \sum_{d \in\{0,1\}^{\bar{V}}}(-1)^{\sum_{v \in V} \sum_{u \in \bar{v}} d_{u}} \prod_{e \in E}\left(\left(\sum_{v \in V} \sum_{u \in \bar{v}} a[\zeta \mid]_{e v} Z_{u} d_{u}\right)-X_{e}\right)
\end{aligned}
$$

and with $c_{v}^{d}:=\sum_{u \in \bar{v}} d_{u} Z_{u} \in C_{\bar{v}}$ further

$$
\begin{aligned}
& =(-1)^{|\bar{V}|} \sum_{d \in\{0,1\}^{\bar{V}}}(-1)^{\sum_{v \in V} c_{v}^{d}(1,1, \ldots, 1)} \prod_{e \in E}\left(\left(\sum_{v \in V} a[\zeta \mid]_{e v} c_{v}^{d}\right)-X_{e}\right) \\
& =(-1)^{|\bar{V}|} \sum_{\left(c_{v}\right) \in \prod_{v \in V} C_{\bar{v}}}(-1)^{\sum_{v \in V} c_{v}(1,1, \ldots, 1)} \prod_{e \in E}\left(\left(\sum_{v \in V} a[\zeta \mid]_{e v} c_{v}\right)-X_{e}\right) \\
& =(-1)^{|\bar{V}|} \sum_{c \in C_{\xi}(Z)}(-1)^{\Sigma \hat{c}} \Pi(A[\zeta \mid] c-X) .
\end{aligned}
$$

Corollary 1.10. Let $\xi: \bar{V} \rightarrow V$ be a square column copier to $A \in R^{E \times V} \supseteq \mathbb{Z}^{E \times V}$.

$$
\operatorname{per}(A[\mid \xi])=(-1)^{|E|} \sum_{c \in \mathbb{N}^{V}}(-1)^{\Sigma c}\left(\begin{array}{c}
\left|\xi^{-1}\right| \\
c
\end{array}\right) \Pi(A c) \quad \text { with } \quad\left(\begin{array}{c}
\left|\xi^{-1}\right| \\
c
\end{array}\right):=\prod_{v \in V}\left(\begin{array}{c}
\left|\xi^{-1}(v)\right| \\
c_{v}
\end{array}\right) .
$$

Proof. We substitute $X=(0,0, \ldots, 0), Z=(1,1, \ldots, 1)$ and $\zeta=\operatorname{Id}_{E}$ in theorem 1.9 . Under this substitution each $c \in C_{\xi}(Z)$ becomes $\hat{c}:=\left(c_{v}(1,1, \ldots, 1)\right)_{v \in V} \in \mathbb{N}^{V}$ and there are exactly $\left(\begin{array}{c}\left|\xi^{-1}\right| \\ \hat{c}\end{array}\right):=\prod_{v \in V}\left(\begin{array}{c}\left|\xi^{-1}(v)\right| \\ \hat{c}_{v}\end{array}\right)$ preimages $c \in C_{\xi}(Z)$ to each $\hat{c} \in \mathbb{N}^{V}$.

This formula shows that if $\operatorname{per}(A[\mid \xi]) \neq 0$ there must be a $c \in \mathbb{N}^{V}$ with $\Pi(A c) \neq 0$ and $\left(\begin{array}{c}\left|\xi^{-1}\right| \\ c^{\prime}\end{array}\right) \neq 0$ i.e. a coloring $c$ of $A$ with $c_{v} \in\left\{0,1, \ldots,\left|\xi^{-1}(v)\right|\right\}$ for all $v \in V$. In order to prove a more general result we need the following lemma. Again $\left|\xi^{-1}\right| !:=$ $\prod_{u \in U}\left(\left|\xi^{-1}(u)\right| !\right)$ for maps $\xi$ into finite sets $U$ : 
Lemma 1.11. Let $\zeta: \bar{E} \rightarrow E$ and $\xi: \bar{V} \rightarrow V$ be a pair of copiers to $A \in R^{E \times V}$ and $\delta: \tilde{V} \rightarrow V$ a copier of the identity matrix $I=I_{V} \in R^{V \times V}$.

The maps $\tilde{\zeta}:=\zeta \uplus \delta: \bar{E} \uplus \tilde{V} \rightarrow E \uplus V$ (with $E$ and $V$ regarded as disjoint) and $\tilde{\xi}:=\xi \uplus \delta: \bar{V} \uplus \tilde{V} \rightarrow V$ form a pair of copiers to $\tilde{A}:=\left(\begin{array}{c}A \\ I\end{array}\right) \in R^{(E \uplus V) \times V}$ and

$$
\operatorname{per}(\tilde{A}[\tilde{\zeta} \mid \tilde{\xi}])=\frac{\left|\tilde{\xi}^{-1}\right| !}{\left|\xi^{-1}\right| !} \operatorname{per}(A[\zeta \mid \xi])
$$

Proof. We prove this by induction on $|\tilde{V}|$. For $\tilde{V}=\varnothing$ the statement holds therefore assume $\tilde{V} \neq \varnothing$ and let $w \in \tilde{V}$ be given. Set $\delta^{\prime}:=\left.\delta\right|_{\tilde{V} \backslash\{w\}}, \tilde{\xi}^{\prime}:=\xi \uplus \delta^{\prime}: \bar{V} \uplus \tilde{V} \backslash\{w\} \rightarrow V$ and $\tilde{\zeta}^{\prime}:=\zeta \uplus \delta^{\prime}: \bar{E} \uplus \tilde{V} \backslash\{w\} \rightarrow E \uplus V$. Laplace expansion of $\tilde{A}[\tilde{\zeta} \mid \tilde{\xi}]$ in the row $w$ yields $\operatorname{per}(\tilde{A}[\tilde{\zeta} \mid \tilde{\xi}])=\left|\tilde{\xi}^{-1}(\delta(w))\right| \cdot \operatorname{per}\left(\tilde{A}\left[\tilde{\zeta}^{\prime} \mid \tilde{\xi}^{\prime}\right]\right)$ since $\tilde{A}[\tilde{\zeta} \mid \tilde{\xi}]$ contains the column $w$ in exactly $\left|\tilde{\xi}^{-1}(\delta(w))\right|$ copies and these are the only columns that are $\neq 0$ (but $=1$ ) in the row $w$. On the other hand $\operatorname{per}\left(\tilde{A}\left[\tilde{\zeta}^{\prime} \mid \tilde{\xi}^{\prime}\right]\right)=\frac{\left|\tilde{\xi}^{\prime-1}\right| !}{\left|\xi^{-1}\right| !} \cdot \operatorname{per}(A[\zeta \mid \xi])$ by the induction hypothesis, proving the statement.

Theorem 1.12 (General color formula). Let $\zeta: \bar{E} \rightarrow E$ and $\xi: \bar{V} \rightarrow V$ be a pair of copiers to $A \in R^{E \times V}, \delta: \tilde{V} \rightarrow V$ an other copier and $Z=\left(Z_{u}\right)_{u \in \bar{V} \uplus \tilde{V}}, \quad X=\left(X_{e}\right)_{e \in \bar{E}}$ and $Y=\left(Y_{u}\right)_{u \in \tilde{V}}$ tuples of indeterminacies.

Set $\tilde{\xi}:=\xi \uplus \delta: \bar{V} \uplus \tilde{V} \rightarrow V, \tilde{v}:=\tilde{\xi}^{-1}(v)$ and $C_{\tilde{v}}:=\left\{\sum_{u \in \tilde{v}} d_{u} Z_{u} \mid d \in\{0,1\}^{\tilde{v}}\right\} \subseteq R[Z]$ for $v \in V$. Let $C_{\tilde{\xi}}(Z):=\left\{c: V \ni v \mapsto c_{v} \in C_{\tilde{v}}\right\}=\prod_{v \in V} C_{\tilde{v}} \subseteq R[Z]^{V}$ be the set of maps that assign an "abstract" color $c_{v}$ from the list $C_{\tilde{v}}$ to each column $v \in V$.

$$
\frac{\left|\tilde{\xi}^{-1}\right| !}{\left|\xi^{-1}\right| !} \operatorname{per}(A[\zeta \mid \xi]) \Pi Z=(-1)^{|\bar{V} \uplus \tilde{V}|} \sum_{c \in C_{\tilde{\xi}}(Z)}(-1)^{\Sigma \hat{c}} \Pi\left(P_{\delta}(c)\right) \Pi(A[\zeta \mid] c-X)
$$

with $P_{\delta}(c):=\left(\Pi\left(c_{v}-Y_{u}\right)_{u \in \delta^{-1}(v)}\right)_{v \in V} \in R[Z, Y]^{V}$ and $\hat{c}:=\left(c_{v}(1,1, \ldots, 1)\right)_{v \in V}$.

Proof. Set $\tilde{X}:=X \uplus Y$ (i.e. $\tilde{X}_{e}=X_{e}$ for $e \in \bar{E}$ and $\tilde{X}_{u}=Y_{u}$ for $\left.u \in \tilde{V}=(\bar{E} \uplus \tilde{V}) \backslash \bar{E}\right)$. With the notation and definitions from lemma 1.11 and color formula 1.9 we have:

$$
\frac{\left|\tilde{\xi}^{-1}\right| !}{\left|\xi^{-1}\right| !} \cdot \operatorname{per}(A[\zeta \mid \xi]) \Pi Z \stackrel{1.11}{=} \operatorname{per}(\tilde{A}[\tilde{\zeta} \mid \tilde{\xi}]) \Pi Z \stackrel{1.9}{=}(-1)^{|\bar{V} \uplus \tilde{V}|} \sum_{c \in C_{\tilde{\xi}}(Z)}(-1)^{\Sigma \hat{c}} \Pi(\tilde{A}[\tilde{\zeta} \mid] c-\tilde{X})
$$

and with $\tilde{\zeta}:=\left.\left.\tilde{\zeta}\right|_{\tilde{V}} \uplus \tilde{\zeta}\right|_{\bar{E}}=\delta \uplus \zeta$ we can evaluate

$$
\Pi(\tilde{A}[\tilde{\zeta} \mid] c-\tilde{X})=\Pi\left(\tilde{A}\left[\left.\tilde{\zeta}\right|_{\tilde{V}} \mid\right] c-Y\right) \cdot \Pi\left(\tilde{A}\left[\left.\tilde{\zeta}\right|_{\bar{E}} \mid\right] c-X\right)=\Pi\left(I_{V}[\delta \mid] c-Y\right) \cdot \Pi(A[\zeta \mid] c-X) .
$$

With $I_{V}=:\left(\partial_{w, v}\right)_{w, v \in V}$ we get $I_{V}[\delta \mid] c \stackrel{1.3}{=}\left(\sum_{v \in V} \partial_{\delta_{u}, v} c_{v}\right)_{u \in \tilde{V}}=\left(c_{\delta_{u}}\right)_{u \in \tilde{V}}$ and can replace

$$
\Pi\left(I_{V}[\delta \mid] c-Y\right)=\Pi\left(c_{\delta_{u}}-Y_{u}\right)_{u \in \tilde{V}}=\Pi\left(\Pi\left(c_{v}-Y_{u}\right)_{u \in \delta^{-1}(v)}\right)_{v \in V}=\Pi\left(P_{\delta}(c)\right) .
$$

Now the substitutions $X=(0,0, \ldots, 0), Z=(1,1, \ldots, 1)$ and $\zeta=\operatorname{Id}_{E}$ (exactly as in the proof of corollary 1.10$)$ yield: 
Corollary 1.13. Let $\xi: \bar{V} \rightarrow V$ be a square column copier to $A \in R^{E \times V}, \delta: \tilde{V} \rightarrow V$ an other copier and $Y=\left(Y_{u}\right)_{u \in \tilde{V}}$ a tuple of indeterminacies. Set $\tilde{\xi}:=\xi \uplus \delta: \bar{V} \uplus \tilde{V} \rightarrow V$.

$$
\frac{\left|\tilde{\xi}^{-1}\right| !}{\left|\xi^{-1}\right| !} \operatorname{per}(A[\mid \xi])=(-1)^{|\bar{V} \uplus \tilde{V}|} \sum_{c \in \mathbb{N}^{V}}(-1)^{\Sigma c}\left(\begin{array}{c}
\left|\tilde{\xi}^{-1}\right| \\
c
\end{array}\right) \Pi\left(P_{\delta}(c)\right) \Pi(A c)
$$

with $P_{\delta}(c):=\left(\Pi\left(c_{v}-Y_{u}\right)_{u \in \delta^{-1}(v)}\right)_{v \in V} \in R[Y]^{V}$ and $\left(\begin{array}{c}\left|\tilde{\xi}^{-1}\right| \\ c\end{array}\right):=\prod_{v \in V}\left(\begin{array}{c}\left|\tilde{\xi}^{-1}(v)\right| \\ c_{v}\end{array}\right)$.

Corollary 1.14. Assume $A \in R^{E \times V} \supseteq \mathbb{Z}^{E \times V}$.

Let color lists $C_{v} \subseteq \mathbb{N}(v \in V)$ with $\sum_{v \in V}\left(\left|C_{v}\right|-1\right)=|E|$ and intervals $M_{v}=$ $\left\{0,1, \ldots, m_{v}\right\} \supseteq C_{v}$ be given. Set $D_{v}:=M_{v} \backslash C_{v}, \tilde{V}:=\biguplus_{v \in V} D_{v}$ and define the copier $\delta: \tilde{V} \rightarrow V$ by $\delta^{-1}(v):=D_{v}$. Let $\xi: \bar{V} \rightarrow V$ be a copier with $\left|\xi^{-1}(v)\right|=\left|C_{v}\right|-1$ and set $\tilde{\xi}:=\xi \uplus \delta: \bar{V} \uplus \tilde{V} \rightarrow V$.

$$
\frac{\left|\tilde{\xi}^{-1}\right| !}{\left|\xi^{-1}\right| !} \operatorname{per}(A[\mid \xi])=(-1)^{|\bar{V} \uplus \tilde{V}|} \sum_{c \in \mathbb{N}^{V}}(-1)^{\Sigma c}\left(\begin{array}{c}
\left|\tilde{\xi}^{-1}\right| \\
c
\end{array}\right) \Pi(P(c)) \Pi(A c)
$$

with $P(c):=\left(\Pi\left(c_{v}-u\right)_{u \in D_{v}}\right)_{v \in V} \in \mathbb{Z}^{V}$.

$P(c)$

Proof. This follows by substituting $Y_{u}=u \in \mathbb{N}$ ( for all $u \in \tilde{V}$ ) in corollary 1.13 .

Now $\operatorname{per}(A[\mid \xi]) \neq 0$ assures the existence of a $c \in \mathbb{N}^{V}$ with $\Pi(A c) \neq 0,\left(\begin{array}{l}\tilde{\xi} \\ c\end{array}\right) \neq 0$ and $P(c) \neq 0 . \quad\left(\begin{array}{c}\left|\tilde{\xi}^{-1}\right| \\ c\end{array}\right) \neq 0$ means $0 \leq c_{v} \leq\left|\tilde{\xi}^{-1}(v)\right|=\left|D_{v}\right|+\left|C_{v}\right|-1=\left|M_{v}\right|-1$ i.e. $c_{v} \in M_{v}$ and $P(c) \neq 0$ means $c_{v} \notin D_{v}$ therefore $c$ is a coloring of $A$ with $c_{v} \in C_{v}$ for all $v \in V:$

Corollary 1.15. Assume $A \in R^{E \times V} \supseteq \mathbb{Z}^{E \times V}$. Let color lists $C_{v} \subseteq \mathbb{N}(v \in V)$ with $\sum_{v \in V}\left(\left|C_{v}\right|-1\right)=|E|$ and a copier $\xi: \bar{V} \rightarrow V$ with $\left|\xi^{-1}(v)\right|=\left|C_{v}\right|-1$ be given.

If $\operatorname{per}(A[\mid \xi]) \neq 0$ then a proper coloring $c: V \ni v \longmapsto c_{v} \in C_{v}$ of $A$ exists.

\section{Graphs}

Notation. In this paper a graph $G$ is a finite multigraph without loops, $V(G)$ denotes its set of vertices, $E(G)$ its edges and $I=I(G): E(G) \longrightarrow\{\{v, w\} \quad$ | $v, w \in V(G), v \neq w\}$, $E(G), I$ $e \longmapsto e^{I}$ its incidence map. ${ }^{I} v:=\left\{e \in E(G) \mid e^{I} \ni v\right\}$ for $v \in V(G)$ and so $\left|{ }^{I} v\right|$ stands for the degree of $v$.

Given a set $C_{v}$ to each element $v$ of a set $V$, an assignment $c: V \ni v \longmapsto c_{v} \in C_{v}$ is a map $c: V \longrightarrow \bigcup_{v \in V} C_{v}, v \longmapsto c_{v}$ with $c_{v} \in C_{v}$ for all $v \in V$.

An assignment of the form $c: V(G) \ni v \longmapsto c_{v} \in C_{v}$ is proper in $e \in E(G)$, if both ends $v_{1}$ and $v_{2}$ of $e$ receive different "colors": $c_{v_{1}} \neq c_{v_{2}}$. It is a (proper vertex)coloring, if it is proper in each edge $e \in E(G)$. We say $G$ is vertex-colorable with n colors if a coloring $c: V(G) \ni v \longmapsto c_{v} \in\{0,1, \ldots, n-1\}$ of $G$ exists.

An assignment of the form $c: E(G) \ni e \longmapsto c_{e} \in C_{e}$ is a (proper edge)-coloring, if every two different incident edges $e, f\left(e^{I} \cap f^{I} \neq \varnothing\right)$ receive different "colors": $c_{e} \neq c_{f}$. 
An assignment $\rightarrow: E(G) \ni e \longmapsto e^{\rightarrow} \in e^{I}$ that to each edge $e \in E(G)$ assigns one of its ends $e^{\rightarrow} \in e^{I} \subseteq V(G)$ is an orientation of $G$. An oriented graph $\vec{G}$ is a Graph $G$ together with an orientation $\rightarrow$ of $G$. In the whole paper $G$ stands for a graph and $\rightarrow$ for an orientation of $G$, we denote its reverse orientation by $\leftarrow\left(e^{I}=\left\{e^{-}, e^{\leftarrow}\right\}\right.$ for all $e \in E(G))$. For vertices $v \in V(G)$ we set $\rightarrow v:=\rightarrow^{-1}(v)=\{e \in E(G) \mid \vec{e}=v\}$ and so $\left|{ }^{\leftarrow} v\right|$ respectively $|\vec{v}|$ stand for the out-respectively indegree of $v \in V(G)$ under $\rightarrow$.

$$
\begin{gathered}
\leftarrow \\
\rightarrow v, \leftarrow v \\
|\rightarrow v|
\end{gathered}
$$

Definition 2.1 (Edge-vertex matrix). Let $V:=V(\vec{G})$ and $E:=E(\vec{G})$.

$$
A(\vec{G})=\left(a_{e v}\right) \in \mathbb{Z}^{E \times V} \quad \text { with } \quad a_{e v}:=\left\{\begin{aligned}
+1 & \text { if } v=e^{-}, \\
-1 & \text { if } v=e^{\leftarrow} \\
0 & \text { otherwise }
\end{aligned}\right.
$$

is the edge-vertex matrix of $\vec{G}$.

\subsection{Orientations and Eulerian subgraphs}

From definitions 1.4 and 2.1 follows:

Proposition 2.2. The orientations $\varphi$ of $\vec{G}$ are exactly the orientations of $A(\vec{G})$ and:

$$
\pi_{A(\vec{G})}(\varphi)=\pi_{\vec{G}}(\varphi):=(-1)^{\left|\left\{e \in E(\vec{G}): e^{\varphi} \neq e^{\vec{x}}\right\}\right|} .
$$

The graph polynomial $f_{\vec{G}}:=\prod_{e \in E(\vec{G})}\left(X_{e^{\rightarrow}}-X_{e^{-}}\right) \in \mathbb{Z}\left[\left(X_{v}\right)_{v \in V(\vec{G})}\right]$ of $\vec{G}$ matches the matrix polynomial (def. 1.7) of $A(\vec{G}): f_{\vec{G}}=f_{A(\vec{G})}$.

Definition 2.3 (Even and odd realizations). An orientation $\varphi$ of $\vec{G}$ is a realization in $\vec{G}$ of a "vertex copier" $\xi: \bar{V} \rightarrow V(\vec{G})$ if it is a realization in $A(\vec{G})$ of $\xi$ as a column copier of $A(\vec{G})$, i.e. if $\left|\varphi_{v}\right|=\left|\xi^{-1}(v)\right|$ for all $v \in V(\vec{G})$. It is an realization of $\delta \in \mathbb{N}^{V}$ if it is a realization of $\delta$ in $A(\vec{G})$, i.e. if $\left|\varphi_{v}\right|=\delta_{v}$ for all $v \in V(\vec{G}) . D_{\delta}(\vec{G}):=D_{\delta}(A(\vec{G}))$ denotes the set of realizations $\varphi$ of $\delta \in \mathbb{N}^{V}$ in $\vec{G} . D E_{\delta}(\vec{G}):=\left\{\varphi \in D_{\delta}(\vec{G}) \mid \pi_{\vec{G}}(\varphi)=1\right\}$ respectively $D O_{\delta}(\vec{G}):=\left\{\varphi \in D_{\delta}(\vec{G}): \pi_{\vec{G}}(\varphi)=-1\right\}$ denotes the set of even respectively odd realizations of $\delta \in \mathbb{N}^{V}$, i.e. the realizations $\varphi$ that are on even respectively odd many edges $e \in E(\vec{G})$ directed opposite to the orientation $\rightarrow$ of $\vec{G}\left(e^{\varphi} \neq e^{\vec{r}}\right)$.

Now corollary 1.6 gives:

Theorem 2.4 (DE-DO-formula). Let $\xi: \bar{V} \rightarrow V(\vec{G})$ be a square copier to $A(\vec{G})$.

$$
\operatorname{per}(A(\vec{G})[\mid \xi])=\left|\xi^{-1}\right| !\left(\left|D E_{\left|\xi^{-1}\right|}(\vec{G})\right|-\left|D O_{\left|\xi^{-1}\right|}(\vec{G})\right|\right)
$$

Especially, $\operatorname{per}(A(\vec{G})[\mid \xi])=0$ if $\xi$ does not have any realizations $\left(D_{\left|\xi^{-1}\right|}(\vec{G})=\emptyset\right)$.

A similar result concerning the coefficients of the graph polynomial was obtained by Alon and Tarsi [AlTa]. In their paper one can also find further infirmation about the existence of orientations and applications. 
Definition 2.5 (Eulerian subgraphs). $\vec{G}$ is called Eulerian if all vertices $v \in V(\vec{G})$ have as many "incoming" as "outgoing" edges: $|\vec{v}|=\left|{ }^{+} v\right| . \quad E u(\vec{G})$ denotes the set of Eulerian subgraphs of $\vec{G}$ (with vertex set $V(\vec{G})$ ). $E E(\vec{G})$ respectively $E O(\vec{G})$ denotes the set of even respectively odd Eulerian subgraphs of $\vec{G}$, i.e. the Eulerian subgraphs with even respectively odd many edges.

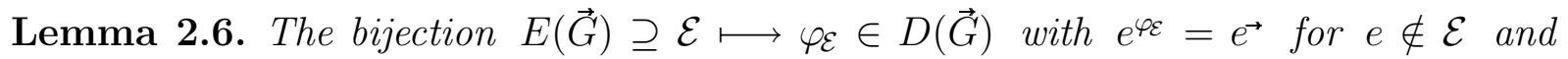
$e^{\varphi_{\mathcal{E}}}=e^{\leftarrow}$ for $e \in \mathcal{E}$ between spanning subgraphs and orientations of $\vec{G}$ can be restricted to bijections $E E(\vec{G}) \rightarrow D E_{\left|\rightarrow^{-1}\right|}(\vec{G})$ and $E O(\vec{G}) \rightarrow D O_{\left|\rightarrow^{-1}\right|}(\vec{G})$.

With this easy to prove lemma (also used in $[\mathrm{AlTa}]$ ) we come from the DE-DOformula 2.4 to the EE-EO-formula:

\section{Theorem 2.7 (EE-EO-formula).}

$$
\operatorname{per}(A(\vec{G})[\mid \rightarrow])=\left|\rightarrow^{-1}\right| !(|E E(\vec{G})|-|E O(\vec{G})|) .
$$

In the case of a bipartite graph $\vec{G}$ we have $E O(\vec{G})=\varnothing$ and $|E E(\vec{G})|-|E O(\vec{G})|$ can be replaced by $|E u(\vec{G})|$. If $\vec{G}$ does not have directed cycles we get $E O(\vec{G})=\varnothing \neq$ $\{\varnothing\}=E E(\vec{G})$ and $\operatorname{per}(A(\vec{G})[\mid \rightarrow])=\left|\rightarrow^{-1}\right|$ ! .

Since the Eulerian subgraphs of $\vec{G}$ are (up to orientation) exactly those of the reverse $\overleftarrow{G}$, we get $\left|\leftarrow^{-1}\right| ! \cdot \operatorname{per}(A(\vec{G})[\mid \rightarrow])=\left|\rightarrow^{-1}\right| ! \cdot \operatorname{per}(A(\overleftarrow{G})[\mid \leftarrow])=(-1)^{|E(\vec{G})|}\left|\rightarrow^{-1}\right| ! \cdot \operatorname{per}(A(\vec{G})[\mid \leftarrow])$ and can deduce the following corollary, which stands here only for completeness:

Corollary 2.8. (Reverse copier) Let $\xi: \bar{V} \rightarrow V(\vec{G})$ be a square column copier to $A(\vec{G})$ with $\left|\xi^{-1}(v)\right| \leq\left|{ }^{I} v\right|$ for all $v \in V(\vec{G})$. Copiers $\xi^{\prime}$ of $A(\vec{G})$ with $\left|\xi^{\prime-1}(v)\right|+\left|\xi^{-1}(v)\right|=\left|{ }^{I} v\right|$ for all $v \in V(\vec{G})$ exist, are square and fulfill

$$
\left|\xi^{-1}\right| ! \operatorname{per}\left(A(\vec{G})\left[\mid \xi^{\prime}\right]\right)=(-1)^{|E(\vec{G})|}\left|\xi^{\prime-1}\right| ! \operatorname{per}(A(\vec{G})[\mid \xi]) .
$$

\subsection{Vertex colorings and the theorem of Alon and Tarsi}

From definitions 1.8 and 2.1 follows:

Proposition 2.9. The colorings $c: V(\vec{G}) \longrightarrow R, v \longmapsto c(v)=c_{v}$ of $\vec{G} \quad\left(c \in R^{V(\vec{G})}\right)$ are exactly the colorings of $A(\vec{G}) \in R^{E \times V}$ and:

$$
A(\vec{G}) c=\left(c\left(e^{\overrightarrow{ }}\right)-c\left(e^{\leftarrow}\right)\right)_{e \in E(\vec{G})} .
$$

This can be combined with the evaluation formulas of section 1.2 to express the permanent $\operatorname{per}(A(\vec{G})[\mid \xi])$ in terms of colorings. With corollary 1.15 follows:

Theorem 2.10 (Permanent condition). Let $C_{v}(v \in V(G))$ be color lists with $\sum_{v \in V}\left(\left|C_{v}\right|-1\right)=|E(\vec{G})| \quad\left(\right.$ e.g. $\left|C_{v}\right|-1=|\vec{v}|$ for all $v \in V$ ) and $\xi: \bar{V} \rightarrow V$ a copier with $\left|\xi^{-1}(v)\right|=\left|C_{v}\right|-1 \quad$ (e.g. $\left.\xi:=\rightarrow\right)$.

If $\operatorname{per}(A(\vec{G})[\mid \xi]) \neq 0$ then a proper coloring $c: V \ni v \longmapsto c_{v} \in C_{v}$ of $\vec{G}$ exists. 
Now the combination with formula 2.7 gives the theorem of Alon and Tarsi [AlTa]:

Theorem 2.11 (Alon, Tarsi 1989). To each $v \in V(\vec{G})$ let $C_{v}$ be a list of $|\vec{v}|+1$ different colors.

If $|E E(\vec{G})| \neq|E O(\vec{G})|$ then a proper coloring $c: V(\vec{G}) \ni v \longmapsto c_{v} \in C_{v}$ of $\vec{G}$ exists.

\subsection{Edge colorings of n-regular graphs}

The edge colorings of a graph are the vertex colorings of its line graph. Therefore, we are especially interested in line graphs. To obtain good results we always assume $G$ to be $n$-regular ( with $n \geq 1$ ).

The vertices of the line graph (interchange graph) $L G$ of a graph $G$ are the edges

$n \geq 1$ of $G, V(L G):=E(G)$. The number of edges between two vertices $v, v^{\prime} \in V(L G)$ in the line graph $L G$ equals the number of common ends of $v$ and $v^{\prime}$ as edges of $G$. If $G$ is $n$-regular then $L G$ is $2(n-1)$-regular, $(n-1)|V(L G)|=|E(L G)|$ and each "vertex copier" $\xi: \bar{V} \longrightarrow V(\overrightarrow{L G})$ to the arbitrary oriented line graph $\overrightarrow{L G}$ of $G$ with $\left|\xi^{-1}(e)\right|=n-1$ for all $e \in V(\overrightarrow{L G})$ is "square" $(|\bar{V}|=|E(\overrightarrow{L \vec{G}})|)$. In this situation the permanent condition 2.10 can be applied and ensures the existence of colorings $c$ : $E(G) \ni e \longmapsto c_{e} \in C_{e}$ to arbitrary lists $C_{e}$ of $n$ different colors if $\operatorname{per}(A(\overrightarrow{L G})[\mid \xi]) \neq 0$.

Since we are especially interested in colorings with equal color lists $C_{e}:=\{0, \ldots, n-1\}$ to the edges $e \in E(G)$ we will now take a closer look at this situation. The line graph $\overrightarrow{L G}$ is partitioned into $|V(G)|$ complete subgraphs $E(v) \subseteq E(\overrightarrow{L G})$, one "around" each vertex $v \in V(G)$ of $G$. If $c: E(G)=V(\overrightarrow{L G}) \longrightarrow\{0,1, \ldots, n-1\}$ is a proper edge coloring of $G$ then the $n$ vertices of each $E(v)$ obtain $n$ different colors under $c$ and thus $\prod_{e \in E(v)}\left(c\left(e^{\rightarrow}\right)-c\left(e^{-}\right)\right)= \pm \prod_{i=1}^{n-1}(i !)$. Now the following definition seems to be useful.

Definition 2.12 (Sign). Let $G$ be a $n$-regular graph, $\overrightarrow{L G}$ its arbitrary oriented line graph. We define the sign respectively the sign in $v \in V(G)$ of an edge coloring $c$ : $E(G)=V(\overrightarrow{L G}) \longrightarrow\{0,1, \ldots, n-1\}$ as follows:

$$
\begin{aligned}
& \operatorname{sign}_{\overrightarrow{L G}}(c, v):=\frac{\prod_{e \in E(v)}\left(c\left(e^{-}\right)-c\left(e^{\leftarrow}\right)\right)}{\prod_{i=1}^{n-1}(i !)} \in\{1,-1\} \quad \text { and } \\
& \operatorname{sign}_{\overrightarrow{L G}}(c):=\prod_{v \in V(G)} \operatorname{sign}_{\overrightarrow{L G}}(c, v) \in\{1,-1\} .
\end{aligned}
$$

With this we obtain a special version of color formula 1.10 (similar results can be found in $[\mathrm{Sch}],[\mathrm{ElGo}])$ :

Theorem 2.13 (Color formula). Let $G$ be a n-regular graph, $\overrightarrow{L G}$ its arbitrary oriented line graph, $\xi$ a copier to $A(\overrightarrow{L G})$ with $\left|\xi^{-1}(v)\right|=n-1$ for all $v \in V(\overrightarrow{L G})$ and $C$ the set of proper edge colorings $c: E(G) \rightarrow\{0,1, \ldots, n-1\}$ of $G$.

$$
\operatorname{per}(A(\overrightarrow{L \vec{G}})[\mid \xi])=\left((-1)^{\frac{n(n-1)}{2}}(n-1) !^{n}\right)^{\frac{1}{2}|V(G)|} \sum_{c \in C} \operatorname{sign}_{\overrightarrow{L G}}(c)
$$


Proof. If there is no proper coloring $(C=\varnothing)$ then $\operatorname{per}(A(\overrightarrow{L G})[\mid \xi])=0$ by theorem 2.10 and the statement holds. In the other case, we have to calculate the different terms in color formula 1.10 with $V:=V(\overrightarrow{L G})=E(G), E:=E(\overrightarrow{L G}), A:=A(\overrightarrow{L G})$. Each color $i \in\{0,1, \ldots, n-1\}$ of a proper edge coloring $c \in C$ occurs on exactly $|V(G)|$ half edges and thus on $\frac{1}{2}|V(G)| \in \mathbb{N}$ edges. Using this we get:

$$
\begin{aligned}
(-1)^{|E|} & =(-1)^{\frac{n(n-1)}{2}|V(G)|}=1 \quad\left(\text { since } \frac{1}{2}|V(G)| \in \mathbb{N}\right), \\
(-1)^{\Sigma c} & =(-1)^{\frac{n(n-1)}{2} \frac{1}{2}|V(G)|}, \\
\left(\begin{array}{l}
\xi \\
c
\end{array}\right)^{2} & =\left(\prod_{i=0}^{n-1} \frac{(n-1) !}{i !(n-1-i) !}\right)^{|V(G)|} \quad(\text { the sets } I v(v \in V(G)) \text { cover } V \text { twice }), \\
\Pi(A c) & \stackrel{2.12}{=}\left(\prod_{i=1}^{n-1}(i !)\right)^{|V(G)|} \cdot \operatorname{sign}_{\overrightarrow{L G}}(c)=\left(\prod_{i=0}^{n-1} i !(n-1-i) !\right)^{\frac{1}{2}|V(G)|} \cdot \operatorname{sign}_{\overrightarrow{L G}}(c), \\
\left(\begin{array}{l}
\xi \\
c
\end{array}\right) \Pi(A c) & =(n-1) ! \frac{n}{2}|V(G)| \cdot \operatorname{sign}_{\overrightarrow{L G}}(c) .
\end{aligned}
$$

Since in color formula 2.13 colorings with different signs may be involved, it can happen that the permanent vanishes $(\operatorname{per}(A(\overrightarrow{L G})[\mid \xi])=0)$ although appropriate edge colorings exist. For example, if there are two different vertices $v$ and $v^{\prime}$ with identical neighborhoods and $n$ is odd this is the case [ElGo, prop. 2.2].

In the planar case, however, all colorings $c: E(G) \longrightarrow\{0,1, \ldots, n-1\}$ have the same sign. This was shown by Ellingham and Goddyn [ElGo, theorem 3.1] in 1994 and generalizes the 3-regular case shown by Vigneron [Vig] in 1946. In 1974 Scheim [Sch] used the older, special version and the graph polynomial to express the number of edge 3-colorings of a planar cubic graph as a permanent. (This case is of special interest since, by the theorem of Tait [JeTo, p.16], the edge 3-colorability of 3-regular planar graphs without bridges is equivalent to the four color theorem of Appel and Haken [ApHa].) With Ellingham and Goddyn's newer result, Scheim's formula can be generalized to $n$-regular planar graphs. In this paper we can deduce this formulas from theorem 2.13:

Theorem 2.14 (Scheim 1974). Let $G$ be a n-regular planar graph, $\overrightarrow{L G}$ its arbitrary oriented line graph, $\xi$ a copier to $A(\overrightarrow{L G})$ with $\left|\xi^{-1}(v)\right|=n-1$ for all $v \in V(\overrightarrow{L G})$ and $C$ the set of proper edge colorings $c: E(G) \rightarrow\{0,1, \ldots, n-1\}$ of $G$.

$$
\operatorname{per}(A(\overrightarrow{L G})[\mid \xi])= \pm(n-1) !^{\frac{n}{2}|V(G)|}|C| \text {. }
$$

Together with theorem 2.10 this leads to Ellingham and Goddyn's partial solution of the list coloring conjecture $[\mathrm{ElGo}]$ :

Theorem 2.15 (Ellingham, Goddyn 1994). Let $G$ be a n-regular planar graph.

If a proper edge coloring $c: E(G) \ni e \longmapsto c_{e} \in\{0,1, \ldots, n-1\}$ of $G$ exists then proper list edge colorings $c: E(G) \ni e \longmapsto c_{e} \in C_{e}$ of $G$ to arbitrary color lists $C_{e}$ of size $n$ also exist. 


\section{Hypergraphs}

Given a matrix $A=\left(a_{e v}\right) \in R^{E \times V}$ we can define a hypergraph $H(A)=\left(V, E, I_{A}\right)$ by

setting $I_{A}:=\left\{(e, v) \in E \times V: a_{e v} \neq 0\right\}$. A map $c: V \longrightarrow R$ is a proper coloring of a hypergraph $H=(V, E, I)$ if every edge is incident with at least two vertices of different color. The following proposition can be easily derived from definition 1.8 .

Proposition 3.1. Let $A=\left(a_{e v}\right) \in R^{E \times V}$ be a matrix with vanishing row sums.

The proper colorings $c: V \longrightarrow R$ of $A$ are proper colorings of $H(A)$.

Conversely, let $S \subseteq R$ be a subring of $R$ (e.g. $R=S\left[X_{1}\right]$ ). If for each row $e \in E$ the equation $\sum_{v \in V} \mu_{v} a_{e v}=0$ with $\left(\mu_{v}\right)_{v \in V} \in S^{V}$ only holds for constant tuples $\left(\mu_{v}\right)_{v \in V}$ then each proper coloring $c: V \longrightarrow S \subseteq R$ of $H(A)$ is a proper coloring of $A$.

\section{References}

[Al] N. Alon: Restricted colorings of graphs. In "Surveys in combinatorics, 1993", London Math. Soc. Lecture Notes Ser. 187, Cambridge Univ. Press, Cambridge 1993, 1-33.

[Al2] N. Alon: Combinatorial Nullstellensatz. Combin. Probab. Comput. 8, No. 1-2 (1999), 7-29.

[AlTa] N. Alon, M. Tarsi: Colorings and orientations of graphs. Combinatorica 12 (1992), 125-134.

[AlTa2] N. Alon, M. Tarsi: A nowhere-zero point in linear mappings. Combinatorica 9 (1989), 393-395.

[ApHa] K. I. Appel, W. Haken, J. Koch: Every planar map is four colorable. Illinois J. Math. 21 (1977), 429-567.

[BrRy] R. A. Brualdi, H. J. Ryser: Combinatorial matrix theory. Cambridge University Press, Cambridge 1991.

[DeV] M. DeVos: Matrix choosability. J. Combin. Theory Ser. A 90 (2000), 197-209.

[ElGo] M. N. Ellingham, L. Goddyn: List edge colourings of some 1-factorable multigraphs. Combinatorica 16 (1996), 343-352.

[JeTo] T. R. Jensen, B. Toft: Graph coloring problems. Wiley, New York 1995.

[Mi] H. Minc: Permanents. Addison-Wesley, London 1978.

[Scha] U. Schauz: Algebraically solvable problems, interpolation over rings and the combinatorial nullstellensatz. In preperation.

[Sch] D. E. Scheim: The number of edge 3-colorings of a planar cubic graph as a permanent. Discrete Math. 8 (1974), 377-382.

[Vig] L. Vigneron: Remarques sur les réseaux cubiques de classe 3 associés au problème des quatre couleurs. C. R. Acad. Sci. Paris T. 223 (1946), 770-772. 